\title{
Asymptotic Behavior of the 3D Compressible Euler Equations with Nonlinear Damping and Slip Boundary Condition
}

\author{
Huimin Yu \\ Department of Mathematics, Shandong Normal University, Jinan 250014, China \\ Correspondence should be addressed to Huimin Yu, hmyu@amss.ac.cn \\ Received 7 January 2012; Accepted 1 March 2012 \\ Academic Editor: Laurent Gosse
}

Copyright (c) 2012 Huimin Yu. This is an open access article distributed under the Creative Commons Attribution License, which permits unrestricted use, distribution, and reproduction in any medium, provided the original work is properly cited.

The asymptotic behavior (as well as the global existence) of classical solutions to the 3D compressible Euler equations are considered. For polytropic perfect gas $\left(P(\rho)=P_{0} \rho^{\gamma}\right)$, time asymptotically, it has been proved by Pan and Zhao (2009) that linear damping and slip boundary effect make the density satisfying the porous medium equation and the momentum obeying the classical Darcy's law. In this paper, we use a more general method and extend this result to the 3D compressible Euler equations with nonlinear damping and a more general pressure term. Comparing with linear damping, nonlinear damping can be ignored under small initial data.

\section{Introduction and Main Results}

We study the 3D compressible Euler equations with nonlinear damping:

$$
\begin{gathered}
\rho_{t}+\nabla \cdot(\rho \vec{u})=0, \\
(\rho \vec{u})_{t}+\nabla \cdot(\rho \vec{u} \otimes \vec{u})+\nabla P(\rho)=-\alpha \rho \vec{u}-\beta \rho|\vec{u}|^{q-1} \vec{u} .
\end{gathered}
$$

This model represents the compressible flow through porous media with nonlinear external force field. Here $\rho, \vec{u}$, and $M=\rho \vec{u}$ denote density, velocity, and momentum, respectively. The pressure $P$ is a smooth function of $\rho$ such that $P(\rho)>0, P^{\prime}(\rho)>0$ for any $\rho>0$. Obviously, for polytropic perfect gas, the pressure term $P(\rho)=P_{0} \rho^{\gamma}\left(P_{0}>0, \gamma>1\right)$ satisfies this condition. $(\nabla \cdot)$ denotes the divergence in $\mathbf{R}^{3}$ and the symbol $\otimes$ denotes the Kronecker tensor product. The external term $-\alpha \rho \vec{u}-\beta \rho|\vec{u}|^{q-1} \vec{u}$ appears in the momentum equation, where $\alpha$ is a positive constant, $\beta$ is another constant but can be either negative or positive. The term $-\alpha \rho \vec{u}$ is called the linear damping and throughout this paper we assume $\alpha \equiv 1$. 
The term $-\beta \rho|\vec{u}|^{q-1} \vec{u}$ with $q>1$ is regarded as a nonlinear source to the linear damping, where the symbol $|\vec{u}|$ denotes $\sqrt{u_{1}^{2}+u_{2}^{2}+u_{3}^{2}}$ if we assume $\vec{u}=\left(u_{1}, u_{2}, u_{3}\right)$. When $\beta>0$, the term $-\beta \rho|\vec{u}|^{q-1} \vec{u}$ is nonlinear damping, while, when $\beta<0$ this term is regarded as nonlinear accumulating. For convenience, we call both the two cases nonlinear damping.

System (1.1) is supplemented by the following initial and boundary conditions:

$$
\begin{gathered}
(\rho, \vec{u})(\vec{x}, 0)=\left(\rho_{0}, \vec{u}_{0}\right)(\vec{x}), \quad \vec{x}=\left(x_{1}, x_{2}, x_{3}\right) \in \Omega, \\
\left.\vec{u} \cdot \vec{n}\right|_{\partial \Omega}=0, \quad t \geq 0, \\
\int_{\Omega} \rho_{0} d \vec{x}=\rho^{*}>0,
\end{gathered}
$$

where $\Omega \in \mathbf{R}^{3}$ is a bounded domain with smooth boundary $\partial \Omega, \vec{n}$ is the unit outward normal vector of $\partial \Omega$, and the last condition is imposed to avoid the trivial case, $\rho \equiv 0$.

For 1D case, system (1.1) and its corresponding $p$-system in Lagrangian coordinates have been studied intensively during the past decades. When $\beta=0$, for various initial and initial-boundary conditions, both the existence and large time behaviors of solutions (including classical and weak solutions) were investigated, see [1-10] and the references therein. [11-13] studied the $p$-system with nonlinear damping $(\beta \neq 0)$. The existence as well as approximate behavior of smooth solutions to the initial boundary condition in half line and Cauchy problem are considered.

From physical point of view, the 3D model (1.1) describes more realistic phenomena. Also, the 3D compressible Euler equations carry some unique features, such as the effect of vorticity, which are totally absent in the 1D case and make the problem more mathematically challenging. Thus, due to strong physical background and significant mathematical challenge, system (1.1) and its time-asymptotic behavior are of great importance and are much less understood than its 1D companion. When $\beta=0$, investigations were carried out among small smooth solutions and we refer the readers to [14-16]. In the direction of nonlinear damping $(\beta \neq 0)$, even the global existence of classical solutions is still open, no less than the asymptotic behavior. In this paper, we consider the global existence and asymptotic behavior of classical solutions to the 3D problem (1.1)(1.2) with nonlinear damping and slip boundary condition.

When $\beta=0$, it has been proved that (see [15]) the dissipation in the momentum equations and the boundary effect make system (1.1) be approximated by the decoupled system

$$
\begin{gathered}
\tilde{\rho}_{t}=\Delta P(\tilde{\rho}), \\
\widetilde{M}=\tilde{\rho} \tilde{\vec{u}}=-\nabla P(\tilde{\rho}),
\end{gathered}
$$

where the first equation is the famous porous medium equation with $P(\rho)=\rho^{\gamma} / \gamma$, while the second one states Darcy's law. The corresponding initial-boundary conditions change into

$$
\begin{gathered}
\tilde{\rho}(\vec{x}, 0)=\tilde{\rho}_{0}(\vec{x}), \\
\left.\nabla P(\tilde{\rho}) \cdot \vec{n}\right|_{\partial \Omega}=0, \quad t \geq 0, \\
\int_{\Omega} \tilde{\rho}_{0} d \vec{x}=\rho^{*}>0 .
\end{gathered}
$$


When $\beta \neq 0$, we will prove in this paper that the effect of nonlinear damping $(\beta<0)$ or accumulating $(\beta>0)$ can be ignored comparing with the linear damping when the initial perturbation around equilibrium state is small.

Before stating our main results, we give some notations. Throughout this paper, $\|\cdot\|$ and $\|\cdot\|_{s}$ denote the norms of $L^{2}(\Omega)$ and $H^{s}(\Omega)$, respectively. For any vector valued function $F=\left(f_{1}, f_{2}, f_{3}\right): \Omega \mapsto \mathbf{R}^{3}$, we denote

$$
\|F\|_{s}^{2}=\left\|f_{1}\right\|_{s}^{2}+\left\|f_{2}\right\|_{s}^{2}+\left\|f_{3}\right\|_{s^{\prime}}^{2} \quad\|F\|_{L^{\infty}}=\left\|f_{1}\right\|_{L^{\infty}}+\left\|f_{2}\right\|_{L^{\infty}}+\left\|f_{3}\right\|_{L^{\infty}}
$$

furthermore, for any functional matrix $M=\left(M_{i j}\right)_{n \times n}: \Omega \rightarrow \mathbf{R}^{\mathbf{n} \times \mathbf{n}}$, we denote

$$
\|M\|_{s}^{2}=\sum_{i, j=1}^{n}\left\|M_{i j}\right\|_{s^{\prime}}^{2} \quad\|M\|_{L^{\infty}}=\sum_{i, j=1}^{n}\left\|M_{i j}\right\|_{L^{\infty}} .
$$

The energy space under consideration is

$$
X_{3}(\Omega,[0, T])=\left\{F: \Omega \times[0, T] \longrightarrow \mathbf{R}^{3}(\text { or } \mathbf{R})\right\}
$$

equipped with norm

$$
\|F\|_{3, T}=\operatorname{ess} \sup _{0 \leq t \leq T}|\|F\||=\operatorname{ess} \sup _{0 \leq t \leq T}\left[\sum_{l=0}^{3}\left\|\partial_{t}^{l} F(\cdot, t)\right\|_{3-l}^{2}\right]^{1 / 2},
$$

for any $F \in X_{3}(\Omega,[0, T])$. The notation $|\Omega|$ denotes the measure of the $\mathbf{R}^{3}$ domain $\Omega$. In this paper, unless specified, $C$ will denote a generic constant which is independent of time. The followings are the main results of this paper.

Theorem 1.1. Suppose the initial data satisfy the compatibility condition $\left.\partial_{t}^{l} \vec{u}(0) \cdot \vec{n}\right|_{\partial \Omega}=0$ for $0 \leq l \leq 2$. Then there exists a constant $\delta>0$ such that if $\left(\rho_{0}-\rho^{*} /|\Omega|, \vec{u}_{0}\right) \in H^{3}(\Omega)$ and $\left\|\left(\rho_{0}-\rho^{*} /|\Omega|, \vec{u}_{0}\right)\right\|_{3} \leq \delta$, the initial boundary condition (1.1) and (1.2) exists a unique global solution $(\rho, \vec{u})$ in $C^{1}(\bar{\Omega} \times[0, \infty)) \cap X_{3}(\Omega,[0, \infty))$. Moreover, the global solution satisfies

$$
\left|\left\|\left(\rho-\frac{\rho^{*}}{|\Omega|}\right)(\cdot, t)\right\|\right|+|\|\vec{u}(\cdot, t)\|| \leq C_{1}\left\|\left(\rho_{0}-\frac{\rho^{*}}{|\Omega|}, \vec{u}_{0}\right)\right\|_{3} \exp ^{-\eta_{1} t}
$$

for some certain positive constants $C_{1}$ and $\eta_{1}$.

Theorem 1.2. Let $(\rho, \vec{u})$ be the unique global smooth solution of $(1.1)$ and $(1.2),(\tilde{\rho}, \tilde{\vec{u}})$ be the global solution of (1.3)(1.4). Then there exist constants $C_{2}$ and $\eta_{2}$ such that

$$
\|(\rho-\tilde{\rho})(\cdot, t)\|_{1}+\|(\vec{u}-\tilde{\vec{u}})(\cdot, t)\| \leq C_{2} \exp ^{-\eta_{2} t}
$$

satisfies for big enough $t>0$. 
Theorem 1.1 states that the global solution of (1.1) and (1.2) converges to the steady state $\left(\rho^{*} /|\Omega|, 0\right)$ exponentially fast in time. To prove this theorem, we first change problem (1.1) into

$$
\begin{gathered}
\rho_{t}+\nabla \cdot(\rho \vec{u})=0, \\
\vec{u}_{t}+\vec{u} \cdot \nabla \vec{u}+\vec{u}+\frac{\nabla P(\rho)}{\rho}=-\beta|\vec{u}|^{q-1} \vec{u}
\end{gathered}
$$

and then we consider the existence and large time behavior of perturbation solution. It is worth pointing out that in [15] the pressure $P$ is assumed to be $P(\rho)=\rho^{\gamma} / \gamma$, then the authors can introduce a nonlinear transformation $\sigma=\left(\rho^{\theta} / \theta\right)(\theta=(\gamma-1) / 2)$ to reformulate the perturbation system as a symmetric hyperbolic system. In this paper, the pressure is more general and the transformation in [15] do not work any more. To overcome this difficulty, we use a symmetrizer to reduce the system to a symmetric hyperbolic one in the sense of Friedrichs. Due to the slip boundary condition, the basic energy estimates can not be applied directly to spatial derivatives. Inspired by $[15,17]$, we use time-derivatives, which still preserve the boundary conditions, to estimate the spatial derivatives.

Theorem 1.2 is our target result. To prove this theorem, we first claim system (1.3) and (1.4) decays to the steady state $\left(\rho^{*} /|\Omega|, 0\right)$ exponentially, too. Then using the triangular inequality we get Theorem 1.2.

Now, we recall some inequalities which will be used in the following.

Lemma 1.3. Let $\Omega$ be any bounded domain in $\mathbf{R}^{3}$ with smooth boundary. Then

$$
\|f\|_{L^{\infty}}(\Omega) \leq C\|f\|_{H^{2}}(\Omega), \quad\|f\|_{L^{p}}(\Omega) \leq C\|f\|_{H^{1}(\Omega)}, \quad 2 \leq p \leq 6
$$

for some constant $C>0$ depending only on $\Omega$.

Lemma 1.4. Let $\vec{u} \in H^{s}(\Omega)$ be a vector-valued function satisfying $\left.\vec{u} \cdot \vec{n}\right|_{\partial \Omega}=0$, where $\vec{n}$ is the unit outer normal of $\partial \Omega$. Then

$$
\|\vec{u}\|_{s} \leq C\left(\|\nabla \times \vec{u}\|_{s-1}+\|\nabla \cdot \vec{u}\|_{s-1}+\|\vec{u}\|_{s-1}\right),
$$

for $s \geq 1$, and the constant $C$ depends only on $s$ and $\Omega$.

\section{Global Existence and Asymptotic Behavior}

In this section, we will consider the global existence and the asymptotic behavior of system (1.11) and (1.2). Due to the boundary effect $(1.2)_{2}$ and the dissipation in the velocity equations, the kinetic energy is conjectured to vanish and the potential energy will converge to a constant as time goes to infinity. Furthermore, since the conservation of mass and the initial condition $(1.2)_{3}$, we expect

$$
(\rho, \vec{u}) \longrightarrow\left(\frac{\rho^{*}}{|\Omega|}, 0\right) \quad \text { as } t \longrightarrow \infty,
$$


where $(\rho, \vec{u})$ is the solution of problem (1.11) and (1.2), and $|\Omega|$ is the measure of $\Omega$ in $\mathbf{R}^{3}$. Without loss of generality, we assume $|\Omega|=1$.

For this purpose, we consider the perturbation system:

$$
\begin{gathered}
\varphi_{t}+\nabla \cdot\left(\left[\varphi+\rho^{*}\right] \vec{u}\right)=0, \\
\vec{u}_{t}+\vec{u} \cdot \nabla \vec{u}+\vec{u}+\frac{\nabla P\left(\left[\varphi+\rho^{*}\right]\right)}{\left[\varphi+\rho^{*}\right]}=-\beta|\vec{u}|^{q-1} \vec{u},
\end{gathered}
$$

where $\varphi=\rho-\rho^{*}$. Let $g(\rho)=Q^{\prime}(\rho)=P^{\prime}(\rho) / \rho$, then $Q^{\prime}(\rho) \nabla \rho=\nabla P(\rho) / \rho$ and system (2.2) changes into

$$
\begin{gathered}
\varphi_{t}+\left[\varphi+\rho^{*}\right] \nabla \cdot \vec{u}+\nabla \varphi \cdot \vec{u}=0, \\
\vec{u}_{t}+\vec{u} \cdot \nabla \vec{u}+\vec{u}+g\left(\varphi+\rho^{*}\right) \nabla \varphi=-\beta|\vec{u}|^{q-1} \vec{u},
\end{gathered}
$$

that is,

$$
\begin{gathered}
\varphi_{t}+\left[\varphi+\rho^{*}\right] \vec{u}_{, i}^{i}+\varphi_{, i} \vec{u}^{i}=0, \\
\vec{u}_{t}^{i}+\vec{u}^{j} \vec{u}_{, j}^{i}+\vec{u}^{i}+g\left(\varphi+\rho^{*}\right) \varphi_{, i}=-\beta|\vec{u}|^{q-1} \vec{u}^{i} .
\end{gathered}
$$

In matrix notation, we have

$$
\begin{aligned}
{\left[\begin{array}{c}
\varphi_{t} \\
\vec{u}_{t}^{1} \\
\vec{u}_{t}^{2} \\
\vec{u}_{t}^{3}
\end{array}\right]+\left[\begin{array}{cccc}
\vec{u}^{1} & \varphi+\rho^{*} & 0 & 0 \\
g\left(\varphi+\rho^{*}\right) & \vec{u}^{1} & 0 & 0 \\
0 & 0 & \vec{u}^{1} & 0 \\
0 & 0 & 0 & \vec{u}^{1}
\end{array}\right]\left[\begin{array}{l}
\varphi, 1 \\
\vec{u}_{1}^{1} \\
\vec{u}_{, 1}^{2} \\
\vec{u}_{, 1}^{3}
\end{array}\right]+\left[\begin{array}{cccc}
\vec{u}^{2} & 0 & \varphi+\rho^{*} & 0 \\
0 & \vec{u}^{2} & 0 & 0 \\
g\left(\varphi+\rho^{*}\right) & 0 & \vec{u}^{2} & 0 \\
0 & 0 & 0 & \vec{u}^{2}
\end{array}\right]\left[\begin{array}{l}
\varphi, 2 \\
\vec{u}_{, 2}^{1} \\
\vec{u}_{, 2}^{2} \\
\vec{u}_{, 2}^{3}
\end{array}\right] } \\
+\left[\begin{array}{cccc}
\vec{u}^{3} & 0 & 0 & \varphi+\rho^{*} \\
0 & \vec{u}^{3} & 0 & 0 \\
0 & 0 & \vec{u}^{3} & 0 \\
g\left(\varphi+\rho^{*}\right) & 0 & 0 & \vec{u}^{3}
\end{array}\right]\left[\begin{array}{l}
\varphi_{, 3} \\
\vec{u}_{, 3}^{1} \\
\vec{u}_{, 3}^{2} \\
\vec{u}_{, 3}^{3}
\end{array}\right]+\left[\begin{array}{c}
0 \\
\vec{u}^{1}+\beta|\vec{u}|^{q-1} \vec{u}^{1} \\
\vec{u}^{2}+\beta|\vec{u}|^{q-1} \vec{u}^{2} \\
\vec{u}^{3}+\beta|\vec{u}|^{q-1} \vec{u}^{3}
\end{array}\right]=0 .
\end{aligned}
$$

Denoting $w=\left[\begin{array}{l}\varphi \\ \vec{u}\end{array}\right]$ and multiplying (2.5) on the left by the symmetrizer

$$
D=\operatorname{diag}\left[g\left(\varphi+\rho^{*}\right), \varphi+\rho^{*}, \varphi+\rho^{*}, \varphi+\rho^{*}\right]
$$

we can rewrite the result to be

$$
D w_{t}+A^{1} w_{, 1}+A^{2} w_{, 2}+A^{3} w_{, 3}+f=0
$$


where

$$
\begin{aligned}
A^{1}= & {\left[\begin{array}{cccc}
g\left(\varphi+\rho^{*}\right) \vec{u}^{1} & \left(\varphi+\rho^{*}\right) g\left(\varphi+\rho^{*}\right) & 0 & 0 \\
\left(\varphi+\rho^{*}\right) g\left(\varphi+\rho^{*}\right) & \left(\varphi+\rho^{*}\right) \vec{u}^{1} & 0 & 0 \\
0 & 0 & \left(\varphi+\rho^{*}\right) \vec{u}^{1} & 0 \\
0 & 0 & 0 & \left(\varphi+\rho^{*}\right) \vec{u}^{1}
\end{array}\right], } \\
A^{2}= & {\left[\begin{array}{cccc}
g\left(\varphi+\rho^{*}\right) \vec{u}^{2} & 0 & \left(\varphi+\rho^{*}\right) g\left(\varphi+\rho^{*}\right) & 0 \\
0 & \left(\varphi+\rho^{*}\right) \vec{u}^{2} & 0 & 0 \\
\left(\varphi+\rho^{*}\right) g\left(\varphi+\rho^{*}\right) & 0 & \left(\varphi+\rho^{*}\right) \vec{u}^{2} & 0 \\
0 & 0 & 0 & \left(\varphi+\rho^{*}\right) \vec{u}^{2}
\end{array}\right], } \\
A^{3}= & {\left[\begin{array}{cccc}
g\left(\varphi+\rho^{*}\right) \vec{u}^{3} & 0 & 0 & \left(\varphi+\rho^{*}\right) g\left(\varphi+\rho^{*}\right) \\
0 & \left(\varphi+\rho^{*}\right) \vec{u}^{3} & 0 & 0 \\
0 & 0 & \left(\varphi+\rho^{*}\right) \vec{u}^{3} & 0 \\
\left(\varphi+\rho^{*}\right) g\left(\varphi+\rho^{*}\right) & 0 & 0 & \left(\varphi+\rho^{*}\right) \vec{u}^{3}
\end{array}\right], } \\
& \left(\varphi+\rho^{*}\right)\left[\begin{array}{cc}
\vec{u}^{1}+\beta|\vec{u}|^{q-1} \vec{u}^{1} \\
\vec{u}^{2}+\beta|\vec{u}|^{q-1} \vec{u}^{2} \\
\vec{u}^{3}+\beta|\vec{u}|^{q-1} \vec{u}^{3}
\end{array}\right] .
\end{aligned}
$$

The existence of global solution to (2.7) can be proved by local existence result and a priori estimates. The local existence is classical and we omit it here. As for the a priori estimates, we first have the following estimate about the temporal derivatives of $w$.

Lemma 2.1. Let $|\|w\|| \leq \delta$ be sufficiently small. Then there exists a positive constant $C_{3}>0$ such that

$$
\frac{d}{d t} \int_{\Omega} \partial_{t}^{k} w \cdot D \partial_{t}^{k} w d \vec{x}+\rho^{*} \int_{\Omega}\left|\partial_{t}^{k} u(\vec{x}, t)\right|^{2} d \vec{x} \leq C_{3}\|w\|^{3},
$$

for $k=0,1,2,3$.

Proof. (1) Zero Order Estimate.

Multiplying (2.7) by $w$ and integrating over $\Omega$, we have

$$
\int_{\Omega} w \cdot D w_{t} d \vec{x}+\sum_{i=1}^{3} \int_{\Omega} w \cdot A^{i} w_{, i} d \vec{x}+\int_{\Omega} w \cdot f d \vec{x}=0
$$


Journal of Applied Mathematics

Using Lemma 1.3 and Cauchy-Schwarz inequality, we have

$$
\begin{aligned}
\int_{\Omega} w \cdot D w_{t} d \vec{x} & =\frac{1}{2} \frac{d}{d t} \int_{\Omega} w \cdot D w d \vec{x}-\frac{1}{2} \int_{\Omega} w \cdot D_{t} w d \vec{x} \\
& \leq \frac{1}{2} \frac{d}{d t} \int_{\Omega} w \cdot D w d \vec{x}+C\left\|D_{t}\right\|_{L^{\infty}} \int_{\Omega}|w|^{2} d \vec{x} \\
& \leq \frac{1}{2} \frac{d}{d t} \int_{\Omega} w \cdot D w d \vec{x}+C|\|w\||^{3} .
\end{aligned}
$$

For the second term coming from the left side of (2.10), we have

$$
\begin{aligned}
\sum_{i=1}^{3} \int_{\Omega} w \cdot A^{i} w_{, i} d \vec{x} & =\frac{1}{2} \int_{\Omega} \sum_{i=1}^{3}\left(w \cdot A^{i} w\right)_{i} d \vec{x}-\frac{1}{2} \int_{\Omega} w \sum_{i=1}^{3} A_{, i}^{i} w d \vec{x} \\
& \leq \frac{1}{2} \int_{\partial \Omega} w \cdot \sum_{i=1}^{3} A^{i} \vec{n}_{i} w d s+C\left\|A_{, i}^{i}\right\|_{L^{\infty}} \int_{\Omega}|w|^{2} d \vec{x}
\end{aligned}
$$

where we have used the Divergence Theorem. Since

$$
w \cdot \sum_{i=1}^{3} A^{i} \vec{n}_{i} w=\left(g \varphi^{2}+2 \rho g \varphi+\rho|\vec{u}|^{2}\right) \vec{n} \cdot \vec{u}=0
$$

Here $g=g(\rho), \rho=\varphi+\rho^{*}$, then (2.12) turns into

$$
\sum_{i=1}^{3} \int_{\Omega} w \cdot A^{i} w_{, i} d \vec{x} \leq C|\|w\||^{3} .
$$

While the term

$$
\int_{\Omega} w \cdot f d \vec{x}=\left(\rho^{*}+\varphi\right) \int_{\Omega}\left(1+\beta|\vec{u}|^{q-1}\right)|\vec{u}|^{2} d \vec{x} \geq \frac{\rho^{*}}{2} \int_{\Omega}|\vec{u}|^{2} d \vec{x}
$$

for $q>1$ and the smallness of $\delta$. Thus, we have

$$
\frac{d}{d t} \int_{\Omega} w \cdot D w d \vec{x}+\rho^{*} \int_{\Omega}|\vec{u}|^{2} d \vec{x} \leq C|\|w\||^{3}
$$

from (2.10) to (2.15). 
(2) Higher Derivatives Estimate.

For $k=1,2,3$, taking $\partial_{t}^{k}$ of system (2.7), we get

$$
D\left\{\partial_{t}^{k} w\right\}_{t}+\sum_{i=1}^{3} A^{i} \partial_{t}^{k} w_{, i}+\partial_{t}^{k} f=-\sum_{l=1}^{k} \frac{k !}{l !(k-l) !}\left\{\partial_{t}^{l} D \partial_{t}^{k-l} w_{t}+\partial_{t}^{l} A^{i} \partial_{t}^{k-l} w_{, i}\right\}
$$

Multiply (2.17) by $\partial_{t}^{k}$ and integrate over $\Omega$ to have

$$
\begin{aligned}
\int_{\Omega} \partial_{t}^{k} w \cdot & D\left\{\partial_{t}^{k} w\right\}_{t} d \vec{x}+\int_{\Omega} \partial_{t}^{k} w \cdot \sum_{i=1}^{3} A^{i} \partial_{t}^{k} w_{, i} d \vec{x}+\int_{\Omega} \partial_{t}^{k} w \cdot \partial_{t}^{k} f d \vec{x} \\
& =-\sum_{l=1}^{k} \frac{k !}{l !(k-l) !} \int_{\Omega}\left\{\partial_{t}^{k} w \cdot \partial_{t}^{l} D \partial_{t}^{k-l} w_{t}+\partial_{t}^{k} w \cdot \partial_{t}^{l} A^{i} \partial_{t}^{k-l} w, i\right\} d \vec{x}
\end{aligned}
$$

Just like the estimates from (2.11) and (2.15), we estimate the three terms on the left side of (2.18) as follows:

$$
\begin{aligned}
\int_{\Omega} \partial_{t}^{k} w \cdot D\left\{\partial_{t}^{k} w\right\}_{t} d \vec{x}= & \frac{1}{2} \frac{d}{d t} \int_{\Omega} \partial_{t}^{k} w \cdot D \partial_{t}^{k} w d \vec{x}-\frac{1}{2} \int_{\Omega} \partial_{t}^{k} w \cdot D_{t} \partial_{t}^{k} w d \vec{x} \\
\leq & \frac{1}{2} \frac{d}{d t} \int_{\Omega} \partial_{t}^{k} w \cdot D \partial_{t}^{k} w d \vec{x}+C\left\|D_{t}\right\|_{L^{\infty}} \int_{\Omega}\left|\partial_{t}^{k} w\right|^{2} d \vec{x} \\
\leq & \frac{1}{2} \frac{d}{d t} \int_{\Omega} \partial_{t}^{k} w \cdot D \partial_{t}^{k} w d \vec{x}+C|\|w\||^{3}, \\
\sum_{i=1}^{3} \int_{\Omega} \partial_{t}^{k} w \cdot A^{i} \partial_{t}^{k} w_{, i} d \vec{x}= & \frac{1}{2} \int_{\Omega} \sum_{i=1}^{3}\left(\partial_{t}^{k} w \cdot A^{i} \partial_{t}^{k} w\right)_{i} d \vec{x}-\frac{1}{2} \int_{\Omega} \partial_{t}^{k} w \cdot \sum_{i=1}^{3} A_{, i}^{i} \partial_{t}^{k} w d \vec{x} \\
\leq & \frac{1}{2} \int_{\partial \Omega} \partial_{t}^{k} w \cdot \sum_{i=1}^{3} A^{i} \vec{n}_{i} \partial_{t}^{k} w d s+C\left\|A_{, i}^{i}\right\|_{L^{\infty}} \int_{\Omega}\left|\partial_{t}^{k} w\right|^{2} d \vec{x} \\
= & \frac{1}{2} \int_{\partial \Omega}\left[g\left(\partial_{t}^{k} \varphi\right)^{2}+\rho\left|\partial_{t}^{k} \vec{u}\right|^{2}\right] \vec{u} \cdot \vec{n} d s+2 \int_{\Omega} \rho g \partial_{t}^{k} \varphi\left(\partial_{t}^{k} \vec{u} \cdot \vec{n}\right) d s \\
& +C|| A_{, i}^{i}\left\|_{L^{\infty}} \int_{\Omega}\left|\partial_{t}^{k} w\right|^{2} d \vec{x} \leq C|\|w\||^{3},\right. \\
\int_{\Omega} \partial_{t}^{k} w \cdot \partial_{t}^{k} f d \vec{x}= & \int_{\Omega} \partial_{t}^{k} \vec{u} \cdot\left(\sum_{l=0}^{k}\left[\partial_{t}^{l}\left(\varphi+\rho^{*}\right) \partial_{t}^{k-l}\left(\vec{u}+\beta|\vec{u}|^{q-1} \vec{u}\right)\right]\right) d \vec{x} \\
\geq & \left(\rho^{*}+\varphi\right) \int_{\Omega}\left(1+\beta|\vec{u}|^{q-1}\right)\left|\partial_{t}^{k} \vec{u}\right|^{2} d \vec{x} \\
& +C \sum_{l=1}^{k} \int_{\Omega} \partial_{t}^{l} \varphi \partial_{t}^{k} \vec{u} \cdot \partial_{t}^{k-l}\left[\left(1+|\vec{u}|^{q-1}\right) \vec{u}\right] d \vec{x} \\
& +C\left(\rho^{*}+\varphi\right) \int_{\Omega} \partial_{t}^{k} \vec{u} \sum_{l=1}^{k} \partial_{t}^{l}\left(1+\beta|\vec{u}|^{q-1}\right) \partial_{t}^{k-l} \vec{u} d \vec{x} \\
\geq & \frac{\rho^{*}}{2} \int_{\Omega}\left|\partial_{t}^{k} \vec{u}\right|^{2} d \vec{x}+C|\|w\||^{3} . \\
& \\
&
\end{aligned}
$$


For the term on the right-hand side of (2.18) we have,

$$
\begin{aligned}
& \quad\left|\int_{\Omega}\left\{\partial_{t}^{k} w \cdot \partial_{t}^{l} D \partial_{t}^{k-l} w_{t}+\partial_{t}^{k} w \cdot \partial_{t}^{l} A^{i} \partial_{t}^{k-l} w_{, i}\right\} d \vec{x}\right| \\
& \quad \leq C\left\|\partial_{t}^{k} w\right\|_{L^{2}}\left\|\partial_{t}^{l} D\right\|_{L^{4}}\left\|\partial_{t}^{k-l} w_{t}\right\|_{L^{4}}+C\left\|\partial_{t}^{k} w\right\|_{L^{2}}\left\|\partial_{t}^{l} A^{i}\right\|_{L^{4}}\left\|\partial_{t}^{k-l} w_{, i}\right\|_{L^{4}} \leq C|\|w\||^{3}
\end{aligned}
$$

for $l=1,2$ and $l \leq k \leq 3$. When $l=k=3$, we get

$$
\begin{aligned}
& \left|\int_{\Omega}\left\{\partial_{t}^{k} w \cdot \partial_{t}^{l} D \partial_{t}^{k-l} w_{t}+\partial_{t}^{k} w \cdot \partial_{t}^{l} A^{i} \partial_{t}^{k-l} w_{, i}\right\} d \vec{x}\right| \\
& \quad \leq C\left\|\partial_{t}^{k} w\right\|_{L^{2}}\left\|\partial_{t}^{l} D\right\|_{L^{2}}\left\|w_{t}\right\|_{L^{\infty}}+C\left\|\partial_{t}^{k} w\right\|_{L^{2}}\left\|\partial_{t}^{l} A^{i}\right\|_{L^{2}}\left\|w_{, i}\right\|_{L^{\infty}} \leq C \mid\|w\| \|^{3} .
\end{aligned}
$$

Combining (2.18) and (2.23), we get

$$
\frac{d}{d t} \int_{\Omega} \partial_{t}^{k} w \cdot D \partial_{t}^{k} w d \vec{x}+\rho^{*} \int_{\Omega}\left|\partial_{t}^{k} u(x, t)\right|^{2} d \vec{x} \leq C|\|w\||^{3}
$$

for $k=1,2,3$. Thus we prove Lemma 2.1.

For slip boundary condition, spatial derivatives can be controlled by temporal derivatives and vorticity that is discussed below.

Lemma 2.2. Let $|\|w\|| \leq \delta$ be sufficiently small and $(\varphi, \vec{u})$ be the solution of (1.11) and (1.2), then there exists a constant $C_{4}$ such that

$$
\|w\|^{2} \leq C_{4}\left(\sum_{l=0}^{2}\left\|\partial_{t}^{l}(\nabla \times \vec{u})\right\|_{2-l}^{2}+\sum_{l=0}^{3}\left\|\partial_{t}^{l} w\right\|^{2}\right) .
$$

Proof. From the velocity equation $(2.3)_{2}$ we have

$$
\nabla \varphi=-\frac{1}{g\left(\rho^{*}+\varphi\right)}\left[\vec{u}+\vec{u}_{t}+\vec{u} \cdot \nabla \vec{u}+\beta|\vec{u}|^{q-1} \vec{u}\right] .
$$

Taking the $L^{2}$ inner product of (2.26) with $\nabla$, we get

$$
\begin{aligned}
\|\nabla \varphi\|^{2} & =-\int_{\Omega} \frac{1}{g\left(\rho^{*}+\varphi\right)}\left[\vec{u}+\vec{u}_{t}+\vec{u} \cdot \nabla \vec{u}+\beta|\vec{u}|^{q-1} \vec{u}\right] \cdot \nabla \varphi d \vec{x} \\
& \leq C\left(\|\vec{u}\|^{2}+\left\|\vec{u}_{t}\right\|^{2}+\|\vec{u}\|_{L^{\infty}}\|\nabla \vec{u}\|^{2}+\beta\|\vec{u}\|_{L^{\infty}}^{q-1}\|\vec{u}\|^{2}\right) \\
& \leq C\left(\|\vec{u}\|^{2}+\left\|\vec{u}_{t}\right\|^{2}\right)+C\|\| w\|\|^{r}
\end{aligned}
$$

where $r=\min \{3, q+1\}>2$. 
The continuity equation $(2.3)_{1}$ implies

$$
\nabla \cdot \vec{u}=-\frac{1}{\varphi+\rho^{*}}\left(\varphi_{t}+\vec{u} \cdot \nabla \varphi\right)
$$

Therefore, we have

$$
\|\nabla \cdot \vec{u}\|^{2} \leq C\left(\left\|\varphi_{t}\right\|^{2}+\|w\|^{3}\right) .
$$

Using Lemma 1.4 with $s=1$, we have

$$
\begin{aligned}
\|\vec{u}\|_{1}^{2} & \leq C\left(\|\nabla \times \vec{u}\|^{2}+\|\nabla \cdot \vec{u}\|^{2}+\|\vec{u}\|^{2}\right) \\
& \leq C\left(\|\nabla \times \vec{u}\|^{2}+\|\vec{u}\|^{2}+\left\|\varphi_{t}\right\|^{2}+\mid\|w\|^{3}\right) .
\end{aligned}
$$

Next, we take time derivatives of (2.26) and (2.28). It is clear that every time derivative up to order two of $\nabla \varphi$ and $\nabla \cdot \vec{u}$ is bounded by $\sum_{l=0}^{3}\left\|\partial_{t}^{l} w\right\|^{2}$. Furthermore, by using an induction on the number of spatial derivatives, we get that the same is true for any derivative up to order of two of $\nabla \varphi$ and $\nabla \cdot \vec{u}$. Applying Lemma 1.4 with $s=1,2,3$, we complete the proof of Lemma 2.2.

From Lemma 2.2, to prove Theorem 1.1 we only need to estimate $\left\|\partial_{t}^{l}(\nabla \times \vec{u})\right\|_{2-l}^{2}$ and $\left\|\partial_{t}^{i} w\right\|^{2}$ for $l=0,1,2, i=0,1,2,3$. The following Lemma is contributed to the estimate of $\nabla \times \vec{u}$.

Lemma 2.3. Let \|\|$w \| \mid \leq \delta$ is sufficiently small, then for any solution $w=(\varphi, \vec{u})$ of problem (1.11)(1.2), one has

$$
\frac{1}{2} \frac{d}{d t}\left(\sum_{l=0}^{2}\left\|\partial_{t}^{l}(\nabla \times \vec{u})\right\|^{2}\right)+\sum_{l=0}^{2}\left\|\partial_{t}^{l}(\nabla \times \vec{u})\right\|^{2} \leq C|\|w(t)\||^{3}
$$

Proof. Taking $\nabla \times$ of the velocity equation in (2.3) and denoting the vorticity $\vec{v}=\nabla \times \vec{u}$, we have

$$
\begin{aligned}
\vec{v}_{t}+\vec{v} & =-\nabla \times(\vec{u} \cdot \nabla \vec{u})-\nabla \times(g(\rho) \nabla \varphi)-\beta \nabla \times\left(|\vec{u}|^{q-1} \vec{u}\right) \\
& =\vec{v} \cdot \nabla \vec{u}-\vec{u} \cdot \nabla \vec{v}-\vec{v} \nabla \cdot \vec{u}-\beta|\vec{u}|^{q-1} \vec{v}-\beta \nabla|\vec{u}|^{q-1} \times \vec{u} .
\end{aligned}
$$

Let $\partial$ denote any mixed time and spatial derivative of order at most 2 , then by taking any mixed derivative of the above equation, we have

$$
\begin{aligned}
\partial \vec{v}_{t}+\partial \vec{v} & =-\partial\left(\nabla \times(\vec{u} \cdot \nabla \vec{u})+\beta \nabla \times\left(|\vec{u}|^{q-1} \vec{u}\right)\right) \\
& =-\partial(-\vec{v} \cdot \nabla \vec{u}+\vec{u} \cdot \nabla \vec{v}+\vec{v} \nabla \cdot \vec{u})-\partial\left(\beta|\vec{u}|^{q-1} \vec{v}+\beta \nabla|\vec{u}|^{q-1} \times \vec{u}\right) .
\end{aligned}
$$


Multiplying the above equation by $\partial \vec{v}$ and integrating the result over $\Omega$, similar the calculations in (2.22) and (2.23), we get

$$
\frac{1}{2} \frac{d}{d t}\|\partial \vec{v}\|^{2}+\|\partial \vec{v}(t)\|^{2} \leq C \mid\|w\|^{3},
$$

by using the Sobolev inequality in Lemma 1.3. This completes the proof of Lemma 2.3.

The next Lemma gives the dissipation in density due to nonlinearity.

Lemma 2.4. There exists a positive constant $C_{5}$ such that

$$
-\frac{d}{d t}\left(\sum_{l=1}^{3} \int_{\Omega}\left(\partial_{t}^{l-1} \varphi \partial_{t}^{l} \varphi\right) d \vec{x}\right)+\sum_{l=0}^{3}\left\|\partial_{t}^{l} \varphi\right\|^{2} \leq C_{5}\left(\|\vec{u}\|^{2}+\left\|\vec{u}_{t}\right\|^{2}+\|w\| \|^{r}\right),
$$

where $r=\min \{3, q+1\}$.

Proof. Due to the conservation of total mass, we know that $\int_{\Omega}\left(\rho-\rho^{*}\right) d \vec{x}=0$, that is, $\int_{\Omega} \varphi d \vec{x}=0$. Using Poincaré's inequality and (2.27), we have

$$
\|\varphi\|^{2} \leq C\|\nabla \varphi\|^{2} \leq C\left(\|\vec{u}\|^{2}+\left\|\vec{u}_{t}\right\|+\left.\|w\|\right|^{r}\right) .
$$

Taking the $t$ derivative of $(2.3)_{1}$ and applying $(\nabla \cdot)$ to $(2.3)_{2}$, we get

$$
\begin{gathered}
\varphi_{t t}+(\nabla \varphi \cdot \vec{u})_{t}+\varphi_{t}(\nabla \cdot \vec{u})+\varphi(\nabla \cdot \vec{u})_{t}=0 \\
(\nabla \cdot \vec{u})_{t}+\nabla \cdot(\vec{u} \cdot \nabla \vec{u})+\nabla \cdot \vec{u}+\nabla \cdot(g(\rho) \nabla \varphi)=-\beta \nabla \cdot\left(|\vec{u}|^{q-1} \vec{u}\right) .
\end{gathered}
$$

$(2.37)-(2.38) \times \varphi$ yields

$$
\varphi_{t t}+(\nabla \varphi \cdot \vec{u})_{t}+\varphi_{t}(\nabla \cdot \vec{u})-\varphi \nabla \cdot\left[\vec{u} \cdot \nabla \vec{u}+g(\rho) \nabla \varphi+\vec{u}+\beta|\vec{u}|^{q-1} \vec{u}\right]=0,
$$

that is,

$$
\varphi_{t t}+(\nabla \varphi \cdot \vec{u})_{t}+\varphi_{t}(\nabla \cdot \vec{u})+\varphi \nabla \cdot \vec{u}_{t}=0
$$

Multiplying (2.40) by $\varphi$, and integrating the result over $\Omega$, we have

$$
\begin{aligned}
& \frac{d}{d t} \int_{\Omega} \varphi \varphi_{t} d \vec{x}-\int_{\Omega} \varphi_{t}^{2} d \vec{x}+\int_{\Omega} \nabla \varphi_{t} \cdot \vec{u} \varphi d \vec{x}+\int_{\Omega} \nabla \varphi \cdot \vec{u}_{t} \varphi d \vec{x} \\
& \quad+\int_{\Omega} \varphi_{t}(\nabla \cdot \vec{u}) \varphi d \vec{x}+\int_{\Omega}\left\{\nabla \cdot\left[\varphi^{2} \vec{u}_{t}\right]-\nabla\left(\varphi^{2}\right) \cdot \vec{u}_{t}\right\} d \vec{x}=0 .
\end{aligned}
$$


Using the Divergence Theorem, we obtain

$$
\begin{aligned}
& \frac{d}{d t} \int_{\Omega} \varphi \varphi_{t} d \vec{x}-\int_{\Omega} \varphi_{t}^{2} d \vec{x}+\int_{\Omega} \nabla \varphi_{t} \cdot \vec{u} \varphi d x+\int_{\Omega} \nabla \varphi \cdot \vec{u}_{t} \varphi d \vec{x} \\
& \quad+\int_{\Omega} \varphi_{t}(\nabla \cdot \vec{u}) \varphi d \vec{x}+\int_{\Omega}\left[\varphi^{2} \vec{u}_{t}\right] \cdot \vec{n} d s-2 \int_{\Omega} \varphi \nabla \varphi \cdot \vec{u}_{t} d \vec{x}=0 .
\end{aligned}
$$

Then we have

$$
-\frac{d}{d t} \int_{\Omega} \varphi \varphi_{t} d \vec{x}+\int_{\Omega} \varphi_{t}^{2} d \vec{x} \leq C|\|w\||^{3}
$$

Furthermore, applying the derivative $\partial_{t}$ to $(2.40)$ we have

$$
\varphi_{t t t}+\left(\nabla \varphi_{t t}\right) \cdot \vec{u}+\nabla \varphi \cdot \vec{u}_{t t}+2 \nabla \varphi_{t} \cdot \vec{u}_{t}+\varphi_{t t}(\nabla \cdot \vec{u})+\varphi_{t}(\nabla \cdot \vec{u})_{t}+\varphi \nabla \cdot \vec{u}_{t t}+\varphi_{t} \nabla \cdot \vec{u}_{t}=0
$$

Taking $L^{2}$ inner product of (2.44) with $\varphi_{t}$, we get

$$
-\frac{d}{d t} \int_{\Omega} \varphi_{t t} \varphi_{t} d \vec{x}+\int_{\Omega} \varphi_{t t}^{2} d \vec{x}-\int_{\Omega} \nabla \cdot\left(\varphi_{t t} \vec{u}+\varphi \vec{u}_{t t}\right) \varphi_{t} d \vec{x}-\int_{\Omega} \nabla \cdot\left(\varphi_{t}^{2} \vec{u}_{t}\right) d \vec{x}-\int_{\Omega} \varphi_{t}^{2}(\nabla \vec{u})_{t} d \vec{x}=0,
$$

that is,

$$
-\frac{d}{d t} \int_{\Omega} \varphi_{t t} \varphi_{t} d \vec{x}+\int_{\Omega} \varphi_{t t}^{2} d \vec{x} \leq C|\|w\||^{3}
$$

where we have used the boundary condition and the Sobolev inequality in Lemma 1.3. Next, apply the derivative $\partial_{t}$ to (2.44) and times the result with $\varphi_{t t}$ to get

$$
-\frac{d}{d t} \int_{\Omega} \varphi_{t t t} \varphi_{t t} d \vec{x}+\int_{\Omega} \varphi_{t t t}^{2} d \vec{x} \leq C|\|w\||^{3}
$$

in which we have used

$$
\varphi_{t t} \nabla \cdot\left(\varphi_{t t t} \vec{u}\right)+\varphi_{t t} \nabla \cdot\left(\varphi \vec{u}_{t t t}\right)=\nabla \cdot\left(\varphi_{t t t} \varphi_{t t} \vec{u}\right)-\nabla \varphi_{t t}\left(\varphi_{t t t} \vec{u}\right)+\nabla \cdot\left(\varphi \varphi_{t t} \vec{u}_{t t t}\right)-\nabla \varphi_{t t}\left(\varphi \vec{u}_{t t t}\right),
$$

and the similar method as (2.46). Combining (2.43), (2.46), and (2.47), we finish the proof of Lemma 2.4. 
Combining Lemmas 2.1 and 2.4, we can characterize the total dissipation of $\sum_{l=0}^{3}\left\|\partial_{t}^{l} w\right\|^{2}$. Let

$$
\begin{gathered}
\lambda=\min \left\{\frac{\rho^{*}}{2 C_{5}}, \frac{\rho^{*}}{4}, \frac{g\left(\rho^{*} / 2\right)}{4}\right\} \\
E(t)=\int_{\Omega}\left(\sum_{l=0}^{3}\left(\partial_{t}^{l} w \cdot D \partial_{t}^{l} w\right)-\lambda \sum_{l=1}^{3}\left(\partial_{t}^{l-1} \varphi \partial_{t}^{l} \varphi\right)\right) d \vec{x} .
\end{gathered}
$$

We can easily see that $\sum_{l=0}^{3}\left\|\partial_{t}^{l} w\right\|^{2}$ and $E(t)$ are equivalent, that is, there exist constants $A$ and $B$ such that

$$
A \sum_{l=0}^{3}\left\|\partial_{t}^{l} w\right\|^{2} \leq E(t) \leq B \sum_{l=0}^{3}\left\|\partial_{t}^{l} w\right\|^{2}
$$

Calculating (2.9) $+\lambda(2.35)$, we have

$$
\frac{d}{d t} \int_{\Omega}\left(\sum_{l=0}^{3}\left(\partial_{t}^{l} w \cdot D \partial_{t}^{l} w\right)-\lambda \sum_{l=0}^{3}\left(\partial_{t}^{l-1} \varphi \partial_{t}^{l} \varphi\right)\right) d \vec{x}+\lambda \sum_{l=0}^{3}\left\|\partial_{t}^{l} w\right\|^{2} \leq C \mid\|w\| \|^{\tilde{r}}
$$

where $\tilde{r}=\min \{3, r\}$, that is,

$$
\frac{d}{d t} E(t)+\frac{\lambda}{B} E(t) \leq C \mid\|w\| \|^{\tilde{r}}
$$

Then Gronwall's inequality and the equivalent relationship (2.50) deduce that

$$
\sum_{l=0}^{3}\left\|\partial_{t}^{l} w\right\|^{2} \leq C \sum_{l=0}^{3}\left\|\partial_{t}^{l} w(0)\right\|^{2} e^{-(\lambda / B) t}+C \int_{0}^{t} e^{s-t}|\|w(s)\||^{\tilde{r}} d s
$$

This inequality combines Lemmas 2.2 and 2.3 deduces the result of Theorem 1.1.

\section{About Porous Medium Equations}

In this section, we investigate the large time behavior of classical solutions to problem (1.3)(1.4). As indicated in the introduction, we expect that (1.1) and (1.2) are captured by (1.3) and (1.4) time asymptotically if

$$
\int_{\Omega} \tilde{\rho}_{0} d \vec{x}=\int_{\Omega} \rho_{0} d \vec{x}=\rho^{*}
$$

Noticing Theorem 1.1 and the triangle inequality, we only need to show that the large time asymptotic state of (1.3) and (1.4) is also the constant state $\left(\rho^{*}, \mathbf{0}\right)$. 
Consider

$$
\begin{gathered}
\tilde{\rho}_{t}=\Delta P(\tilde{\rho}), \\
\tilde{\rho}(\vec{x}, 0)=\tilde{\rho}_{0}(x), \\
\left.\nabla P(\tilde{\rho}) \cdot \vec{n}\right|_{\partial \Omega}=0, \quad t \geq 0,
\end{gathered}
$$

with the initial data

$$
\int_{\Omega} \tilde{\rho}_{0} d \vec{x}=\rho^{*}, \quad \tilde{\rho}_{0}(\vec{x}) \in L^{\infty}(\Omega) .
$$

Here we assume $\tilde{\rho}_{0}$ is uniform bounded, that is, there exists a constant $\rho^{*}<\bar{\rho}<\infty$ such that

$$
0 \leq \tilde{\rho}_{0}(\vec{x}) \leq \bar{\rho}
$$

The global existence and the large time behavior of solutions to (3.2) and (3.3) have been established in [18]. The method we used in this section is similar with which in [15]. Yet, since the pressure is more general in this paper, we can not use the corresponding result in [15] directly.

Lemma 3.1. Let $\tilde{\rho}$ be the global solution of problem (3.2) and (3.3), $\widetilde{M}=-\nabla P(\tilde{\rho})$. Then there exist positive constants $C>0, \eta>0$, independent of time $t$, such that

$$
\left\|\left(\tilde{\rho}-\rho^{*}\right)\right\|_{1}+\|\widetilde{M}\| \leq C e^{-\eta t}
$$

for big enough $t$.

Proof. From [18], we know that there exists a positive constant $T>0$ such that the problem (3.2) and (3.3) has a classical solution $\tilde{\rho}(\vec{x}, t)$ for $t>T$, and

$$
\tilde{\rho}(\vec{x}, t)>\frac{\rho^{*}}{2}, \quad \text { for } t>T \text {. }
$$

On the other hand, due to the comparison principle

$$
0 \leq \tilde{\rho}(\vec{x}, t) \leq \bar{\rho}, \quad \text { for any }(\vec{x}, t) \in \bar{\Omega} \times\left[0, L^{\infty}\right)
$$

For $t>T$, We consider

$$
\left(\tilde{\rho}-\rho^{*}\right)_{t}=\Delta\left(P(\tilde{\rho})-P\left(\rho^{*}\right)\right) .
$$


Journal of Applied Mathematics

Taking $L^{2}$ inner product of (3.8) with $\left(\tilde{\rho}-\rho^{*}\right)$, we have

$$
\begin{aligned}
0 & =\frac{1}{2} \frac{d}{d t} \int_{\Omega}\left(\tilde{\rho}-\rho^{*}\right)^{2} d \vec{x}-\int_{\Omega} \Delta\left(P(\tilde{\rho})-P\left(\rho^{*}\right)\right)\left(\tilde{\rho}-\rho^{*}\right) d \vec{x} \\
& =\frac{1}{2} \frac{d}{d t}\left\|\tilde{\rho}-\rho^{*}\right\|^{2}+\int_{\Omega} \frac{\left|\nabla\left(P(\tilde{\rho})-P\left(\rho^{*}\right)\right)\right|^{2}}{P^{\prime}(\tilde{\rho})} d \vec{x},
\end{aligned}
$$

where we have used boundary condition $(3.2)_{3}$. Combining the increasing property of $P^{\prime}(\widetilde{\rho})$ and the inequality (3.7), we have

$$
\frac{1}{2} \frac{d}{d t}\left\|\tilde{\rho}-\rho^{*}\right\|^{2}+\frac{1}{P^{\prime}(\bar{\rho})}\left\|\nabla\left(P(\tilde{\rho})-P\left(\rho^{*}\right)\right)\right\|^{2} \leq 0 .
$$

Multiplying (3.2) 1 with $P^{\prime}(\tilde{\rho})$, we have

$$
P(\tilde{\rho})_{t}-P^{\prime}(\tilde{\rho}) \Delta P(\tilde{\rho})=0,
$$

that is,

$$
\left(P(\tilde{\rho})-P\left(\rho^{*}\right)\right)_{t}-P^{\prime}(\tilde{\rho}) \Delta\left(P(\tilde{\rho})-P\left(\rho^{*}\right)\right)=0 .
$$

Define

$$
\psi=\tilde{\rho}-\rho^{*}, \quad \phi=P(\tilde{\rho})-P\left(\rho^{*}\right) .
$$

Taking $L^{2}$ inner product of (3.12) with $\Delta \phi$, we get

$$
\frac{1}{2} \frac{d}{d t}\|\nabla \phi\|^{2}+P^{\prime}\left(\frac{\rho^{*}}{2}\right)\|\Delta \phi\|^{2} \leq 0
$$

(3.10) plus (3.14) deduce

$$
\frac{1}{2} \frac{d}{d t}\left(\|\psi\|^{2}+\|\nabla \phi\|^{2}\right)+\tilde{C}\left(\|\nabla \phi\|^{2}+\|\Delta \phi\|^{2}\right) \leq 0,
$$

where $\tilde{C}=\min \left\{P^{\prime}\left(\rho^{*} / 2\right), 1 / P^{\prime}(\bar{\rho})\right\}$. Since the conservation of total mass and the Poincaré's inequality, we have

$$
\|\psi\|^{2} \leq C\|\nabla \psi\|^{2} \leq C\left(\frac{1}{P^{\prime}\left(\rho^{*} / 2\right)}\right)^{2}\|\nabla \phi\|^{2} .
$$

Then inequality (3.15) turns into

$$
\frac{1}{2} \frac{d}{d t}\left(\|\psi\|^{2}+\|\nabla \phi\|^{2}\right)+C\left(\|\psi\|^{2}+\|\nabla \phi\|^{2}+\|\Delta \phi\|^{2}\right) \leq 0
$$

The Gronwall inequality and (3.16) deduce Lemma 3.1. 


\section{Acknowledgments}

This project is supported by the National Natural Science Foundation of China (Grant no. 10901095) and the Promotive Research Fund for Excellent Young and Middle-Aged Scientists of Shandong Province (Grant no. BS2010SF025).

\section{References}

[1] C. M. Dafermos, "A system of hyperbolic conservation laws with frictional damping," Zeitschrift für Angewandte Mathematik und Physik, vol. 46, pp. 294-307, 1995.

[2] C. M. Dafermos and R. Pan, "Global BV solutions for the $p$-system with frictional damping," SIAM Journal on Mathematical Analysis, vol. 41, no. 3, pp. 1190-1205, 2009.

[3] X. X. Ding, G. Q. Chen, and P. Z. Luo, "Convergence of the fractional step Lax-Friedrichs scheme and Godunov scheme for the isentropic system of gas dynamics," Communications in Mathematical Physics, vol. 121, no. 1, pp. 63-84, 1989.

[4] L. Hsiao and T. P. Liu, "Convergence to nonlinear diffusion waves for solutions of a system of hyperbolic conservation laws with damping," Communications in Mathematical Physics, vol. 143, no. 3, pp. 599-605, 1992.

[5] F. M. Huang, P. Marcati, and R. H. Pan, "Convergence to the Barenblatt solution for the compressible Euler equations with damping and vacuum," Archive for Rational Mechanics and Analysis, vol. 176, no. 1, pp. 1-24, 2005.

[6] F. M. Huang and R. H. Pan, "Asymptotic behavior of the solutions to the damped compressible Euler equations with vacuum," Journal of Differential Equations, vol. 220, no. 1, pp. 207-233, 2006.

[7] F. M. Huang and R. H. Pan, "Convergence rate for compressible Euler equations with damping and vacuum," Archive for Rational Mechanics and Analysis, vol. 166, no. 4, pp. 359-376, 2003.

[8] K. Nishihara and T. Yang, "Boundary effect on asymptotic behaviour of solutions to the $p$-system with linear damping," Journal of Differential Equations, vol. 156, no. 2, pp. 439-458, 1999.

[9] K. Nishihara, W. Wang, and T. Yang, "Lp-convergence rate to nonlinear diffusion waves for $p$-system with damping," Journal of Differential Equations, vol. 161, no. 1, pp. 191-218, 2000.

[10] R. Pan, "Darcy's law as long-time limit of adiabatic porous media flow," Journal of Differential Equations, vol. 220, no. 1, pp. 121-146, 2006.

[11] C. K. Lin, C. T. Lin, and M. Mei, "Asymptotic behavior of solution to nonlinear damped $p$-system with boundary effect," International Journal of Numerical Analysis and Modeling B, vol. 1, no. 1, pp. 70-92, 2010.

[12] M. Mei, "Nonlinear diffusion waves for hyperbolic $p$-system with nonlinear damping," Journal of Differential Equations, vol. 247, no. 4, pp. 1275-1296, 2009.

[13] C. T. Zhu and M. N. Jiang, " $L^{p}$-decay rates to nonlinear diffusion waves for $p$-system with nonlinear damping," Science in China A, vol. 49, no. 6, pp. 721-739, 2006.

[14] T. C. Sideris, B. Thomases, and D. H. Wang, "Long time behavior of solutions to the 3D compressible Euler equations with damping," Communications in Partial Differential Equations, vol. 28, no. 3-4, pp. 795-816, 2003.

[15] R. Pan and K. Zhao, "The 3D compressible Euler equations with damping in a bounded domain," Journal of Differential Equations, vol. 246, no. 2, pp. 581-596, 2009.

[16] W. Wang and T. Yang, "The pointwise estimates of solutions for Euler equations with damping in multi-dimensions," Journal of Differential Equations, vol. 173, no. 2, pp. 410-450, 2001.

[17] Y. Guo and W. Strauss, "Stability of semiconductor states with insulating and contact boundary conditions," Archive for Rational Mechanics and Analysis, vol. 179, no. 1, pp. 1-30, 2006.

[18] J. L. Vázquez, The Porous Medium Equation, The Clarendon Press Oxford University Press, Oxford, UK, 2007. 


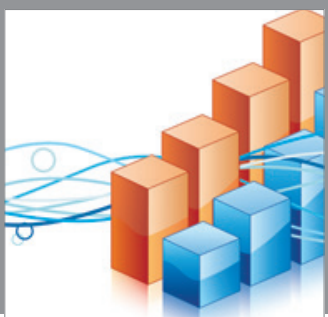

Advances in

Operations Research

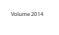

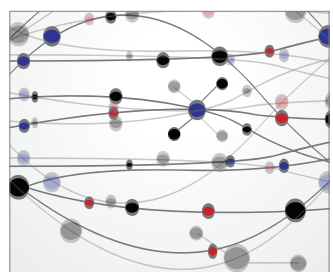

\section{The Scientific} World Journal
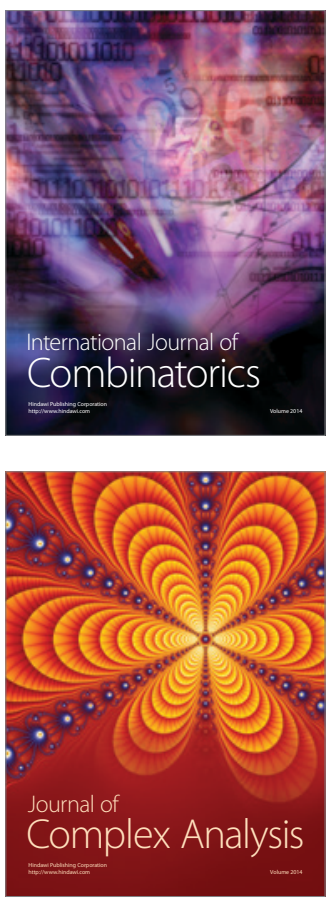

International Journal of

Mathematics and

Mathematical

Sciences
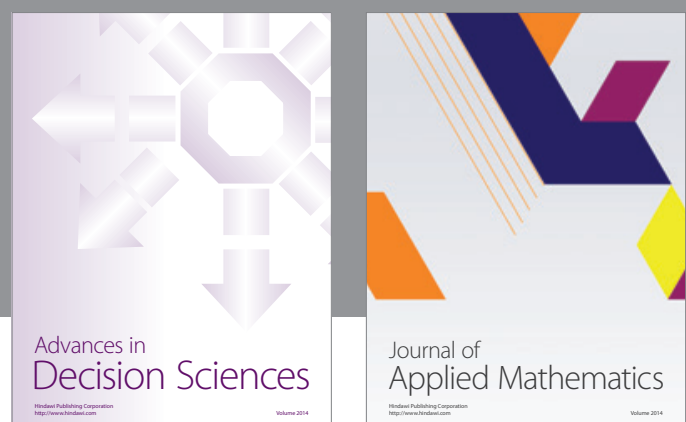

Journal of

Applied Mathematics
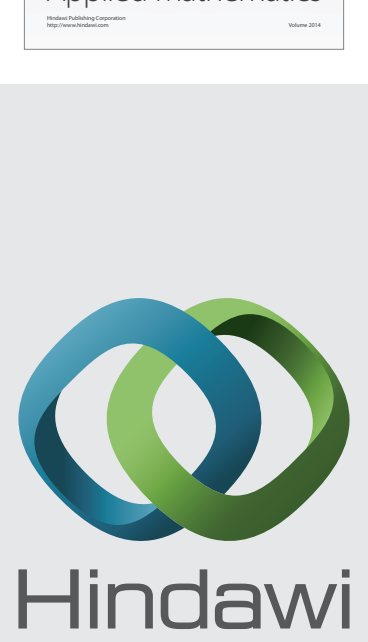

Submit your manuscripts at http://www.hindawi.com
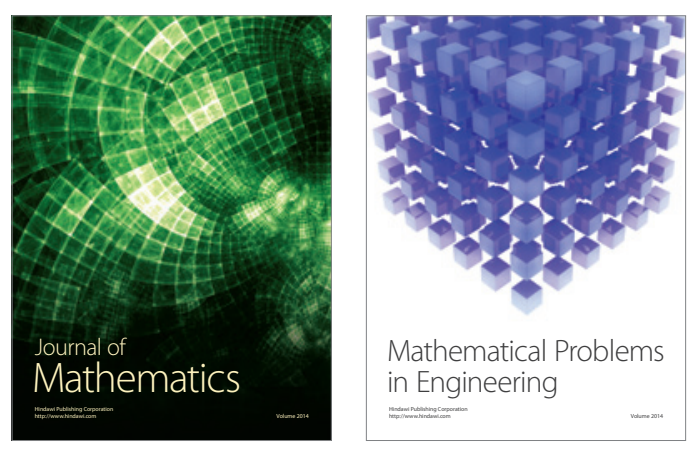

Mathematical Problems in Engineering
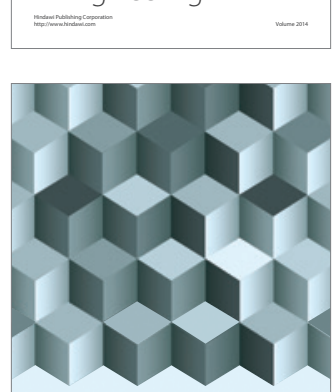

Journal of

Function Spaces
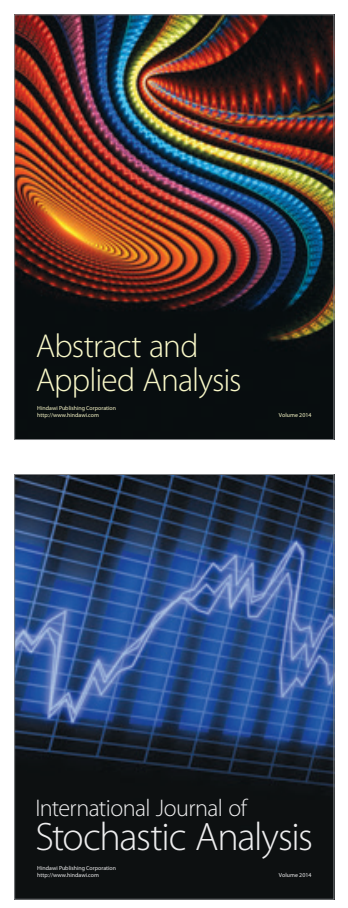

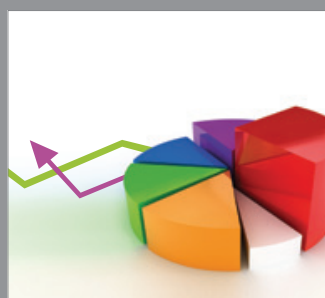

ournal of

Probability and Statistics

Promensencen
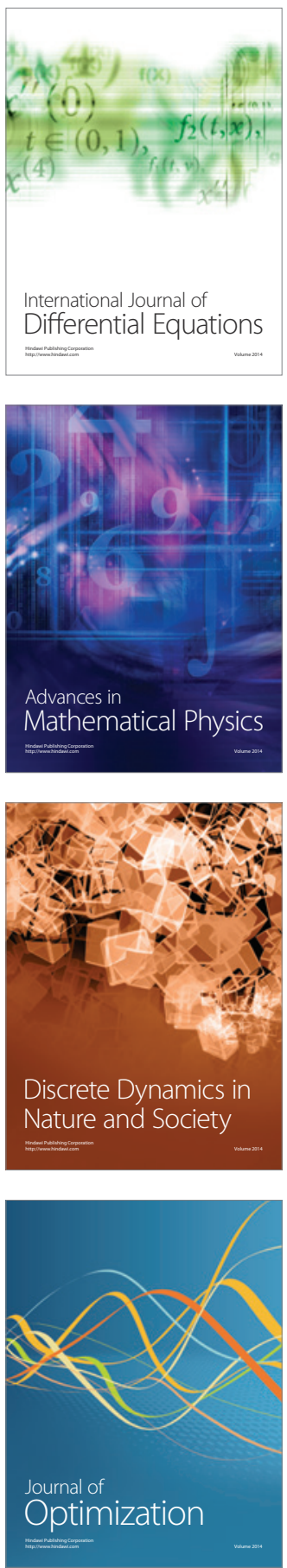